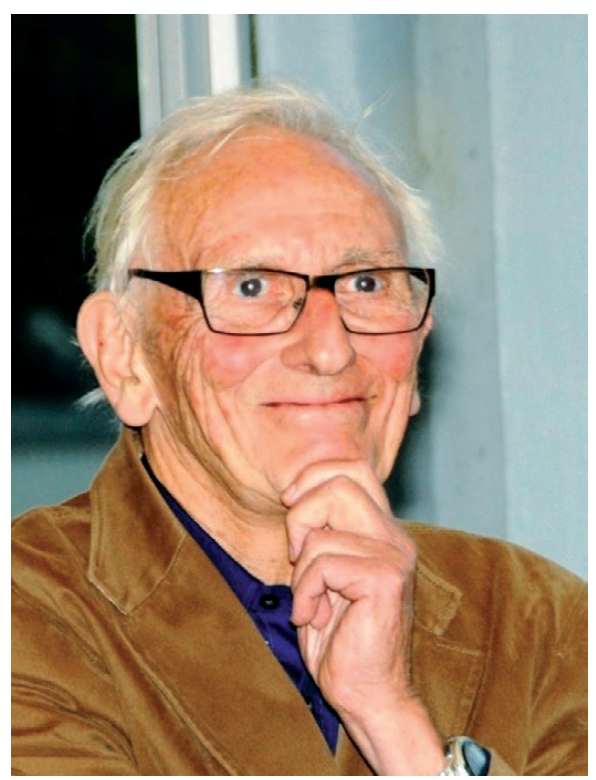

\title{
Roger Maynard : un grand physicien, qui a mis ses talents au service de la communauté scientifique
}

Roger Maynard, président de la Société Française de Physique de 2005 à 2007, et professeur émérite à l'Université Joseph Fourier de Grenoble depuis 2003, est décédé le 30 mai 2015.

Roger Maynard est né à Nice en 1938. Après avoir effectué ses études de physique à l'Université de Marseille, il s'est installé en 1961 au Polygone scientifique Louis Néel à Grenoble, afin de commencer une thèse sur la physique de la matière condensée à basse température, sous la responsabilité du professeur Dreyfus. Depuis, il n'a plus jamais quitté ce lieu, à l'exception d'un séjour postdoctoral à Los Angeles.

En tant que scientifique, Roger Maynard est l'auteur d'une centaine de publications internationales, quelques-unes avec un indice de citation largement supérieur à 200. Toujours avec une intuition originale et innovatrice, il s'est intéressé à de nombreuses thématiques : les verres, les phonons, l'optique non linéaire, la mésoscopie des photons, la supraconductivité et la nanophysique, l'acoustique, les cristaux liquides, la théorie de l'information, la sismologie, la physique numérique... Une vingtaine de thèses ont été rédigées sous sa responsabilité. Il a soutenu un nombre incalculable de jeunes chercheurs, nombre qui dépasse probablement la centaine. Roger Maynard a été organisateur et animateur d'une vingtaine de grandes conférences et écoles internationales, aux Houches, à Cargèse, à Paris. L'école qu'il a organisée aux Houches en août 1978 avec Roger Balian et Gérard Toulouse sur la "Matière Mal Condensée » - avec l'intégration de nombreux (futurs) prix Nobel au sein des jeunes doctorants - a probablement été l'une des meilleurs écoles de physique jamais organisées, avec une formule qui a été exemplaire pour celles qui ont suivi. Roger Maynard a très activement contribué à la création d'une interface pluridisciplinaire entre la physique de la matière condensée et d'autres domaines en physique, comme l'optique et la géophysique, dont profitera encore très longtemps la nouvelle génération.

Roger Maynard a été précurseur en tant qu'enseignant et administrateur. Il a fondé l'un des deux premiers Magistères de la Physique en 1986, avec l'École normale supérieure de Paris, puis a créé l'Association des Responsables de Magistère en France et porté le projet de la Maison des Magistères de l'Université Joseph Fourier sur le Polygone scientifique de Grenoble. C'est là-bas qu'il a fondé en 1990 la jeune équipe "Expérimentation Numérique ", qu'il a dirigé jusqu'à sa reconnaissance en 1998 en tant qu'unité mixte de recherche de l'Université de Grenoble 1 et du CNRS. Le "Laboratoire de Physique et Modélisation des Milieux Condensés UMR 5493 " héberge actuellement de nombreux (jeunes) chercheurs et enseignants-chercheurs, et est devenu un laboratoire d'une grande excellence scientifique, porteur de la physique théorique et numérique à Grenoble.

Dans les années 1990, Roger Maynard s'est impliqué dans l'administration universitaire. De 1994 à 1998, il a été vice-président de l'Université Joseph Fourier, chargé de la recherche, dans l'équipe de Daniel Bloch. Entre 1999 et 2002, il a occupé différentes fonctions de conseiller (nanosciences) auprès du ministère de la Recherche. Autour de son $65^{\mathrm{e}}$ anniversaire, en février 2003, la communauté scientifique internationale lui a rendu hommage, et plus de 150 scientifiques se sont manifestés à Grenoble, y compris des dirigeants de l'Université, du CNRS et du ministère de la Recherche. Début 2005, c'est-à-dire pendant l'Année mondiale de la physique, Roger Maynard a été élu président de la Société Française de Physique, une responsabilité qu'il exercera jusqu'à l'année 2007. Pendant les cinq années suivantes, il a présidé le Conseil d'administration d'EDP Sciences, l'éditeur scientifique français, dont les actionnaires sont les sociétés savantes de chimie (SCF), de physique (SFP), d'optique (SFO) et de mathématiques appliquées (SMAI). Jusqu'à sa mort, Roger Maynard était un membre très actif du Comité d'Éthique du CNRS (COMETS). À ce titre, il est intervenu régulièrement sur les publications scientifiques, le plagiat, les archives ouvertes.

Sa toute dernière passion scientifique était la flèche du temps. Encore tout récemment, fin avril 2015, il a fait une intervention grand public dans la section locale de la SFP à Rennes, intervention qui est postée en ligne sur YouTube.

Bart Van Tiggelen et Alain Fontaine, pour le Conseil d’administration de la Société Française de Physique 


\section{Les engagements de Roger Maynard}

Roger Maynard nous a quittés brusquement le 30 mai dernier. Un petit nombre de ses proches le savait affecté par une implacable maladie de la moelle, qui affaiblissait ses défenses immunitaires. Mais tous ses collègues et amis ont été pris par surprise par cette terrible nouvelle, tant Roger était resté actif, plein d'entrain et de projets jusqu'à ses derniers jours.

On savait Roger très passionné par ses recherches. Je voudrais apporter ici un témoignage sur d'autres activités auxquelles il consacrait beaucoup de son temps avec générosité. Il eut un rôle fondamental à la Mission Scientifique Universitaire au début des années 2000 : chargé d'établir les budgets de recherche de toutes les universités françaises en physique et sciences pour l'ingénieur, il fut à la fois admiré et craint pour la rigueur de sa vision. C'est à cette période aussi qu'il eut l'intuition prémonitoire de l'importance des nanosciences et lança la première Action Concertée Incitative du domaine, grâce à quelques maigres fonds que nous avions détournés de concert à partir d'autres programmes ministériels.

Nos activités communes se sont poursuivies pendant les années de sa présidence de la SFP, suivie de la mienne, fortement marquées par la loi relative aux Libertés et Responsabilités des Universités. Nous avons ensemble accompagné les innovations dans la politique universitaire par des analyses et des mises en garde, parfois écoutées. Une autre grande affaire fut le lancement par la SFP des Écoles de Physique Avancée au Maghreb, qui connurent des réalisations dans chacun des trois pays. La SFP, grâce à Roger, a tissé des liens très étroits avec la Société Tunisienne de Physique : c'est à Carthage qu'il fit l'une de ses dernières brillantes conférences sur l'irréversibilité du Temps, dans une école de métrologie coorganisée avec nos amis tunisiens.

Enfin Roger fut très actif au Comité d'éthique du CNRS, où nous nous sommes retrouvés en 2011. L'ouverture des sciences au monde et à la population le passionnait. Il a beaucoup contribué à la réflexion du CNRS sur les publications en "open access gold", pour lesquelles sa connaissance de l'édition chez EDP Sciences l'incitait à la prudence. Au sujet de la science dite citoyenne, il insistait toujours sur la nécessaire diffusion de la culture scientifique auprès de tous les publics, et aussi sur l'importance d'être à l'écoute de la population sur les sujets sensibles qui suscitent des controverses.

C'était un bonheur de travailler avec Roger : il était attentif, curieux de la pensée des autres, prêt au consensus, mais pourtant ferme sur certains principes d'un rationalisme de bon aloi. Sa gentillesse et sa patience étaient sans mesure. Sa disparition est pour beaucoup d'entre nous une perte que nous n'avons pas fini de mesurer.

Michèle Leduc

\section{Du Bulletin de la SFPà Reflets de la physique}

C'est au printemps 2005 que j'ai rencontré pour la première fois Roger Maynard, nouvellement élu président de la SFP, alors que je venais de prendre la responsabilité de la rédaction du Bulletin de la SFP. Roger était convaincu de l'importance, pour la Société Française de Physique, du maintien d'une revue sur papier comme vecteur de rubriques et d'articles approfondis et réfléchis (à côté d'un site internet rénové, de réactivité élevée). Mais il souhaitait que le Bulletin, dont le premier numéro datait de 1970 [1] et dont la présentation n'avait pas beaucoup évolué depuis, soit profondément rénové, avec des articles scientifiques plus courts et d'une pédagogie plus travaillée, une forme plus attractive, et une plus grande ouverture aux débats de science et société et aux problèmes de politique scientifique. Roger pouvait être très persuasif : il m'a rapidement convaincu. C'est encore lui qui, à l'automne de la même année, plutôt que de se contenter d'une simple évolution du Bulletin, a pris la décision de faire appel pour la réalisation de cette revue rénovée, à des maquettistes professionnels sélectionnés à la suite d'un appel d'offre. Laetitia Morin et Keith Villemeur conçurent alors et mirent au point la maquette actuelle.

Enfin, c'est toujours Roger qui décida de changer le nom de la revue, pour lequel nous avons consulté une soixantaine de responsables de la SFP. Le premier numéro de Reflets de la physique parut ainsi en octobre 2006, avec un éditorial de Roger Maynard.

Charles de Novion 